\title{
MENSURAÇÃO DE HORAS DE TRABALHO DO ENFERMEIRO EM HOSPITAL FILANTRÓPICO TERCIÁRIO
}

Fernanda Novaes Moreno ${ }^{1}$, Maria do Carmo Lourenço Haddad², Marli Terezinha Oliveira Vannuchi², Sabine Jenal ${ }^{3}$, Suellen Karina de Oliveira Girotti ${ }^{4}$

\begin{abstract}
RESUMO: Estudo quantitativo, exploratório e transversal objetivou mensurar o tempo empregado em atividades desempenhadas por enfermeiros de um hospital filantrópico terciário. Os dados foram coletados em 2010 por meio da observação direta e sistematizada do tempo despendido em atividades assistenciais, gerenciais e de apoio desses profissionais, durante 120 horas. Verificou-se que durante a jornada de trabalho os enfermeiros consumiram $39 \%$ do tempo em atividades administrativas, $33 \%$ em atividades assistenciais e $28 \%$ em atividades de apoio ou relacionadas ao atendimento a terceiros. A contribuição deste estudo se deu através do reconhecimento do papel desempenhado pelo enfermeiro durante a jornada de trabalho, o que possibilitou visualizar as lacunas entre suas atribuições legais e o processo de trabalho real, além de favorecer a gerência do dimensionamento desse profissional para a clientela atendida, a partir do diagnóstico situacional de como esse investe o tempo durante a jornada de trabalho.

PALAVRAS-CHAVE: Gerência; Serviços de enfermagem; Jornada de trabalho; Papel do profissional de enfermagem.
\end{abstract}

\section{MEASURING NURSES’ WORK HOURS IN A PHILANTHROPIC TERTIARY HOSPITAL}

ABSTRACT: This quantitative, exploratory and transversal study aimed to measure the time employed in activities carried out by nurses in a philanthropic tertiary hospital. The data was collected in 2010 by systematic direct observation of the time spent in care activities, management activities, and in support of nurses and managers, during a period of 120 hours. It was ascertained that during the workday the nurses spent $39 \%$ of the time on administrative activities, 33\% on care activities, and $28 \%$ in support activities or in attending to third parties. The contribution of this study lies in its recognition of the role fulfilled by the nurses during the workday, which made it possible to identify the gaps between they are legally expected to do and the real work process, besides benefitting the management of training and equipping these professionals for the clients attended, based on a situational diagnosis of how they invest their time during the workday. KEYWORDS: Management; Nursing services; Workday; Role of the nursing professional.

\section{MENSURACIÓN DE HORAS DE TRABAJO DEL ENFERMERO EN HOSPITAL FILANTRÓPICO TERCIARIO}

RESUMEN: Estudio cuantitativo, exploratorio y transversal cuyo objetivo fue mensurar el tiempo empleado en actividades de enfermeros de un hospital filantrópico terciario. Los datos fueron recogidos en 2010 por medio de la observación directa y sistematizada del tiempo gastado en actividades asistenciales, administrativas y de apoyo de eses profesionales, durante 120 horas. Se ha verificado que durante la jornada laboral los enfermeros consumieron 39\% del tiempo en actividades administrativas, $33 \%$ en actividades asistenciales y $28 \%$ en actividades de apoyo o relacionadas al atendimiento a terceros. La contribución de este estudio se muestra por medio del reconocimiento del papel del enfermero durante su jornada de trabajo, lo que posibilitó visualizar las lagunas entre sus atribuciones legales y el proceso de trabajo real, además de favorecer la administración con fines de dimensionar ese profesional para la clientela atendida, a partir del diagnóstico situacional de como ese invierte su tiempo durante su periodo de trabajo.

PALABRAS-CLAVE: Administración; Servicios de enfermería; Periodo de trabajo; Papel del profesional de enfermería.

\footnotetext{
${ }^{1}$ Enfermeira. Mestranda pelo Programa Pós-Graduação em Enfermagem da Universidade Estadual de Londrina -UEL. Professora do Departamento de Enfermagem do Centro de Ciências da Saúde da UEL.

${ }^{2}$ Enfermeira. Doutora em Enfermagem. Professora do Departamento de Enfermagem do Centro de Ciências da Saúde da UEL.

${ }^{3}$ Enfermeira. Mestranda pelo Programa de Pós-Graduação em Enfermagem da Escola de Enfermagem de Ribeirão Preto da Universidade de São Paulo. Gerente Multiprofissional da Irmandade da Santa Casa de Londrina.

${ }^{4}$ Enfermeira. Mestranda pelo Programa de Pós-Graduação em Enfermagem da UEL. Enfermeira da Comissão de Controle de Infecção Hospitalar da Irmandade Santa Casa de Londrina.
}

Autor correspondente:

Fernanda Novaes Moreno

Universidade Estadual de Londrina

Rua Robert Koch, 60 - 86038-440 - Londrina-PR-Brasil

E-mail: ferzinhaenf@hotmail.com
Recebido: $15 / 06 / 2011$

Aprovado: 03/01/2012

Cogitare Enferm. 2012 Jan/Mar; 17(1):50-6 


\section{INTRODUÇÃO}

Estudar as práticas profissionais dos enfermeiros é um tema cuja discussão deve ser valorizada e intensificada devido à necessidade de conhecer as principais atividades realizadas no âmbito hospitalar, a fim de analisar se estas práticas correspondem às necessidades dos pacientes.

Desde 1986 o planejamento da assistência de enfermagem é uma imposição legal no Brasil. De acordo com a Lei do Exercício Profissional n. 7498, o enfermeiro exerce todas as atividades de enfermagem, cabendolhe, privativamente, o planejamento, organização, coordenação e avaliação dos serviços de assistência de enfermagem $^{(1)}$. Reforçando a importância e necessidade de se planejar a assistência de enfermagem, a Resolução do Conselho Federal de Enfermagem (COFEN) n. 272/2002, artigo n. 2, afirma que a implementação da Sistematização da Assistência de Enfermagem (SAE) deve ocorrer em toda instituição da saúde, seja pública ou privada ${ }^{(2)}$. É por meio do planejamento que o enfermeiro define, com antecedência, as ações e os cuidados a serem realizados pela equipe de enfermagem para atender as necessidades dos pacientes.

O enfermeiro é o profissional responsável pela prática gerencial, a quem compete à coordenação da equipe de técnicos e auxiliares de enfermagem, condução e viabilização do processo de cuidado $^{(3)}$; esta prática também contribui para a qualidade da assistência prestada, pois este profissional direciona as prioridades e os cuidados necessários ao paciente de forma digna, segura e ética.

Para compreensão das práticas dos enfermeiros, é necessário conhecer a definição desta profissão para relacioná-la com as atividades desenvolvidas por eles. Autores $^{(4: 96-101)}$ afirmam que:

(...) enfermagem é uma das profissões da área da saúde cuja essência e especificidade é o cuidado ao ser humano individualmente, na família ou na comunidade, desenvolvendo atividade de promoção, prevenção de doenças, recuperação e reabilitação de saúde, atuando em equipes, seja coordenando outros setores para a prestação da assistência e promovendo a autonomia dos pacientes através da educação em saúde.

O enfermeiro possui quatro atividades que norteiam a sua profissão: assistencial, gerencial, educativa e de pesquisa. Essas atividades não podem ser realizadas separadamente, pois a ligação entre elas é um fator importante para prestar assistência de enfermagem com segurança ${ }^{(3)}$.

Em um documento da Organização Mundial da Saúde, o qual apresenta as descrições da Enferma- gem em todo o mundo, demonstra que a qualificação do pessoal de enfermagem e suas atividades diferem profundamente de um local para o outro; conclui que a natureza e a prática da profissão são influenciadas pela realidade que compreende a política, a economia e a cultura local ${ }^{(4)}$.

O conhecimento das práticas desenvolvidas pelos enfermeiros no cotidiano das instituições é de fundamental importância, pois possibilita identificar as lacunas existentes no processo de trabalho e, consequentemente, auxiliar na delimitação de suas funções. Além disso, pode trazer elementos que contribuam para um desempenho criativo, inovador, participativo que atenda as necessidades do paciente e do trabalhador.

Assim, este estudo teve por objetivo mensurar o tempo despendido em atividades desempenhadas por enfermeiros de um hospital terciário filantrópico situado no norte do Paraná.

\section{MÉTODO}

Trata-se de um estudo quantitativo, exploratório e transversal realizado em um hospital filantrópico terciário situado no Norte do Paraná. A instituição possui 221 leitos e média de internação de 860 pacientes por mês; desde 2005 tem sido implantada a SAE com quatro fases - histórico, exame físico, evolução e prescrição - das seis proposta por Horta ${ }^{(5)}$. A jornada de trabalho dos enfermeiros é de 42 horas semanais em turnos diurnos de 6 horas e plantões nos finais de semana e, no turno noturno, esses profissionais cumprem jornada de 12 horas de trabalho por 36 horas de descanso.

Participaram do estudo 23 enfermeiros assistenciais, que foram categorizados quanto ao sexo e experiência profissional em anos e que atuam nas unidades de Pronto Atendimento (PA), Centro de Emergência e Trauma (CET), Unidades de Terapia Intensiva (UTI 1 e UTI3) e Unidades de Internação (Unidade 2, Unidade 3, Unidade 4, Unidade 5 e Unidade 6), sendo escolhidos aleatoriamente por turnos manhã, tarde e noite, tendo como único critério de inclusão atuar como enfermeiro assistencial na instituição há mais de seis meses.

A coleta de dados foi realizada por alunos do sétimo semestre de enfermagem de uma universidade conveniada com a instituição sediadora, os quais foram previamente capacitados para a realização da pesquisa por uma das pesquisadoras. Os dados foram coletados nos meses de agosto a setembro de 2010, totalizando 120 horas de observação.

Utilizou-se a técnica de observação direta e sistema- 
tizada das atividades desenvolvidas pelos enfermeiros e registrou-se o tempo despendido em cada procedimento, sendo os procedimentos classificados em 9 tarefas assistenciais, 4 gerenciais e 11 de apoio. Os dados provenientes da observação direta foram registrados em um roteiro estruturado desenvolvido pela gerência de enfermagem, embasados nas necessidades gerenciais da instituição, que continha questões referentes às atividades assistenciais, gerenciais e de apoio.

Institucionalmente considera-se como atividades assistenciais as funções desempenhadas diretamente com ou para o paciente, ou seja, os cuidados diretos prestados pelo enfermeiro, como também os cuidados indiretos, sob supervisão do enfermeiro, porém executados pelo profissional técnico, tais como: higiene e conforto, auxílio ao paciente na alimentação, realização de curativos, verificação de sinais vitais, atendimento às solicitações de pacientes, entre outros.

Para as atividades gerenciais considera-se aquelas referentes ao planejamento, organização, comando, coordenação e controle das atividades desenvolvidas pela equipe de enfermagem.

As atividades de apoio foram consideradas aquelas que não demandam assistência direta ou indireta ao paciente, como atendimento a visita de familiares, informações a terceiros, equipe médica e serviço de nutrição, atendimento ao telefone, passagem de plantão, atendimento à supervisão, orientações aos funcionários, conferência de materiais, solicitação de serviço de manutenção e atendimento à auditoria.

O tempo das atividades observadas foi verificado em minutos e a ocorrência da saturação das informações obtidas foi utilizada como critério para a delimitação da coleta de dados. Desta forma, foi possível identificar com fidedignidade o conteúdo das atividades realizadas pelos enfermeiros da instituição.

As atividades foram codificadas em tabelas demonstrativas, utilizando o Programa Excel for Windows $2007^{\circledR}$, visando à melhor compreensão da dimensão e característica das ações.

Este estudo deriva do projeto de pesquisa intitulado "Grau de Dependência de clientes internados em Hospital Filantrópico de Alta Complexidade", aprovado pelo Comitê de Ética em Pesquisa da Instituição, tendo sido obtido parecer favorável com registro no Sistema Nacional de Informação sobre Ética em Pesquisa, CAAE $n^{\circ}$ 0026.0.083.083-10.

No período do estudo estavam sendo realizadas pesquisas institucionais concomitantes, em relação ao dimensionamento de pessoal e carga de trabalho, esta coleta de dados auxiliou no levantamento estatístico das atividades desenvolvidas pelo enfermeiro a fim de categorizar as atividades prioritárias e buscar dimensionar dentro da instituição qual seria o número ideal de enfermeiros para determinada população e como potencializar o turno de trabalho para melhor qualidade da assistência.

\section{RESULTADOS}

Dos 23 enfermeiros observados, 20(87\%) eram do sexo feminino e $3(13 \%)$ do sexo masculino; $16(70 \%)$ atuavam no turno diurno, $6(26 \%)$ à noite e $1(4 \%)$ em período integral. Quanto ao tempo de atuação na instituição, 10(43\%) trabalhavam há mais de quatro anos, $6(26 \%)$ entre um a dois anos, $4(17 \%)$ atuavam há mais de seis meses e $3(13 \%)$ entre três a quatro anos. Da amostra estudada, $17(74 \%)$ profissionais exerciam suas atividades em Unidades de Internação, 3(13\%) em Pronto Socorro e 3(13\%) em Unidade de Terapia Intensiva.

Com base na observação da realidade e pela cronometragem do tempo utilizado para a realização das atividades assistenciais, gerenciais e de apoio foram categorizadas durante seis horas do plantão, que serão demonstrados a seguir.

A média de tempo, em minutos e por unidade de estudo, entre as atividades assistenciais foi: 3,2 para higiene e conforto; 5,1 no auxílio ao paciente na alimentação; 5,2 na realização de curativos; 13,9 na verificação de sinais vitais; 2,4 no atendimento às solicitações de pacientes; 29,6 na realização de visita de enfermagem; 18,8 em atendimento à intercorrências; 7,4 na admissão e alta e 17 minutos no preparo de pacientes para exames. A média geral de tempo dedicado pelo enfermeiro para a assistência direta ao paciente foi de 97 minutos em 6 horas de trabalho, ou seja, aproximadamente um terço do tempo (Tabela 1).

A média de tempo, em minutos e por unidade de estudo, entre as atividades gerenciais foi: 82,7 na realização da SAE; 10 na checagem de prontuário; 15 no preenchimento de documentos e 8,1 minutos para armazenar prontuários. Esses dados mostram que mesmo com dispêndio baixo de tempo os enfermeiros utilizam parte do tempo realizando atividades que supostamente poderiam ser delegadas a pessoal técnico administrativo, como a escriturária. Foram verificadas quatro atividades gerenciais e observou-se que a SAE foi a mais realizada, com tempo médio de 82,7 minutos no turno de 6 horas (Tabela 2).

A média de tempo, em minutos e por unidade 
Tabela 1 - Média de tempo, em minutos, das atividades assistenciais realizadas pelos enfermeiros. Londrina, 2010

\begin{tabular}{lccccccccc}
\hline & U2 & U3 & U4 & U5 & U6 & PA & CET & UTI-1 & UTI-3 \\
\hline Higiene e conforto & 6,7 & 1,2 & 4,0 & 5,0 & 3,6 & 0,0 & 3,1 & 5,8 & 0,0 \\
Auxílio na alimentação & 2,0 & 5,0 & 5,3 & 16,7 & 2,5 & 4,5 & 3,6 & 2,5 & 4,5 \\
Realização de Curativo & 7,2 & 2,0 & 13,8 & 2,4 & 6,0 & 2,5 & 1,0 & 0,0 & 12,1 \\
Verificação de Sinais Vitais & 8,3 & 2,4 & 6,3 & 1,6 & 4,7 & 4,2 & 8,6 & 39,6 & 50,1 \\
Atendimento às solicitações depacientes & 0,0 & 7,0 & 5,9 & 2,5 & 0,0 & 3,3 & 1,0 & 0,0 & 2,0 \\
Visita de enfermagem & 27,4 & 41,2 & 59,0 & 35,2 & 23,3 & 9,5 & 15,5 & 18,4 & 37,6 \\
Atendimento à intercorrência & 11,4 & 22,1 & 22,16 & 10,7 & 7,2 & 8,2 & 8,2 & 15,6 & 19,1 \\
Admissão e alta & 11,8 & 16,0 & 13,7 & 3,0 & 8,0 & 0,0 & 5,2 & 9,3 & 0,0 \\
Preparo de paciente para exames & 23,1 & 15,0 & 16,4 & 19,7 & 20,0 & 12,3 & 10,2 & 14,9 & 21,6 \\
$\quad$ TOTAL & 98,2 & 112,0 & 146,5 & 97,0 & 75,6 & 44,6 & 56,5 & 106,2 & 147,1 \\
\hline \multicolumn{1}{c}{$\quad$} & & & & & & & &
\end{tabular}

Tabela 2 - Média de tempo, em minutos, das atividades gerenciais realizadas por enfermeiros. Londrina, 2010

\begin{tabular}{lccccccccc}
\hline & U2 & U3 & U4 & U5 & U6 & PA & CET & UTI-1 & UTI-3 \\
\hline SAE* & 55,1 & 94,9 & 98,4 & 95,8 & 73,3 & 65,5 & 61,3 & 84,7 & 116,1 \\
Checar prontuário & 8,7 & 18,1 & 11,2 & 11,7 & 3,3 & 4,3 & 5,4 & 13,2 & 14,5 \\
Preencher documentos & 9,8 & 12,0 & 19,0 & 39,5 & 9,2 & 11,4 & 10,0 & 11,1 & 13,5 \\
Armazenar prontuários & 5,1 & 6,7 & 5,7 & 7,1 & 9,2 & 10,0 & 2,0 & 13,9 & 13,5 \\
$\quad$ TOTAL & 78,8 & 131,7 & 124,3 & 153,7 & 95,0 & 91,2 & 87,1 & 122,9 & 157,6 \\
\hline *SAE- Sistematizacão da Assistência de Enfermagm & & & & & & & &
\end{tabular}

SAE- Sistematização da Assistência de Enfermagem

de estudo, entre as atividades de apoio foi: 28,3 no atendimento ao telefone; 20,6 com informações a terceiros, 13,3 na orientação para as visitas de pacientes; 11 com informações a nutricionista, 10,4 para passagem de plantão para outro turno; $9,9 \mathrm{com}$ informações a equipe médica; 7,3 com informações para auditoria; 7,2 com orientações ao funcionário; 5,4 com informações a supervisão; 2,8 com armazenamento de material e 0,9 minutos com solicitação de serviço de manutenção (Tabela 3). Observa-se que o tempo consumido pelas enfermeiras no atendimento ao telefone, média de 28.3 minutos, é superior à soma do tempo gasto com a troca de informações entre outros profissionais. As ações que caracterizaram o atendimento ao telefone consistem em: passagem de plantão para admissão do paciente no caso de alta ou transferência entre setores, comunicação da lista de dietas, ligações para médicos e outros profissionais envolvidos com o atendimento ao paciente, agendamento de horário no Centro Cirúrgico, solicitações de vagas em UTI, entre outros.

Verifica-se na tabela 4 que, durante a jornada de trabalho, os enfermeiros conseguem manter um equilíbrio entre as atividades que lhes competem, sendo que a atividade gerencial demanda um pouco mais de tempo, considerando ser esta privativa ao enfermeiro.

Tabela 3 - Média de tempo, em minutos, das atividades de apoio realizadas por enfermeiros. Londrina,2010

\begin{tabular}{lccccccccc}
\hline & U2 & U3 & U4 & U5 & U6 & PA & CET & UTI-1 & UTI-3 \\
\hline Visitas de familiares & 6,2 & 14,4 & 21,5 & 13,6 & 17,1 & 3,7 & 8,2 & 20,8 & 14,3 \\
Informações a terceiros & 11,2 & 19,7 & 17,8 & 12,0 & 16,7 & 22,5 & 25,9 & 31,4 & 28,7 \\
Atendimento de telefone & 22,3 & 22,8 & 20,9 & 12,8 & 18,5 & 21,4 & 79,5 & 24,4 & 32,9 \\
Informações ao médico & 9,1 & 9,0 & 10,8 & 8,6 & 8,5 & 7,8 & 11,3 & 16 & 8,5 \\
Informações a nutrição & 16,3 & 14,8 & 10,5 & 3,0 & 9,8 & 5,0 & 6,2 & 12,5 & 21,1 \\
Passagem de plantão & 12,3 & 11,8 & 11,0 & 10 & 17,8 & 7,2 & 4,7 & 9,3 & 10,0 \\
Atendimento a supervisão & 3,2 & 9,5 & 6,3 & 11,2 & 0 & 0 & 0 & 7,5 & 11,5 \\
Orientação ao funcionário & 7,3 & 7,0 & 6,3 & 16,0 & 4,5 & 15,0 & 2,5 & 5,3 & 1,5 \\
Conferência de material & 3,5 & 1,5 & 2,0 & 3,8 & 4,5 & 2,5 & 3,2 & 4,5 & 0,0 \\
Solicitação de manutenção & 2,5 & 6,0 & 0,0 & 0,0 & 0,0 & 0,0 & 0,0 & 0,0 & 0,0 \\
Atendimento a auditoria & 10,0 & 5,0 & 8,0 & 12,0 & 5,0 & 0,0 & 0,0 & 12,0 & 14,0 \\
\multicolumn{1}{r}{ TOTAL } & 104,0 & 121,7 & 115,2 & 103,1 & 102,7 & 85,2 & 141,6 & 143,8 & 142,6 \\
\hline
\end{tabular}


Tabela 4 - Tempo total das atividades assistencial, gerencial e de apoio consumido por enfermeiros. Londrina, 2010

\begin{tabular}{lccccccccc}
\hline \multicolumn{1}{c}{ Atividade } & \multicolumn{2}{c}{ Manhã } & \multicolumn{2}{c}{ Tarde } & \multicolumn{2}{c}{ Noite } & \multicolumn{3}{c}{ Total em 24 horas } \\
& $\%$ & min. & $\%$ & min. & $\%$ & min. & $\%$ & Minutos & Horas \\
\hline Assistencial & 33 & 118,8 & 29 & 104,4 & 37 & 129,6 & 33 & 475,2 & $08 \mathrm{~h} 31 \mathrm{~min}$ \\
Gerencial & 37 & 133,2 & 41 & 147,6 & 38 & 136,8 & 39 & 561,6 & $09 \mathrm{~h} 35 \mathrm{~min}$ \\
Atendimento / apoio & 30 & 108 & 30 & 108 & 25 & 93,6 & 28 & 403,2 & $07 \mathrm{~h} 11 \mathrm{~min}$ \\
$\quad$ TOTAL & 100 & 360 & 100 & 360 & 100 & 360 & 100 & 1440 & $24 \mathrm{horas}$ \\
\hline
\end{tabular}

\section{DISCUSSÃO}

Entre as atividades assistenciais executadas pelos enfermeiros observa-se que realizar visitas de enfermagem despendeu maior tempo dos enfermeiros. Essa atividade é relevante visto que é parte integrante do processo de planejamento, permite aumentar o vínculo com os pacientes, detectar e prevenir problemas através de um exame físico direcionado e coletar dados individualizados oferecendo, principalmente, subsídios para avaliar a assistência prestada ao cliente ${ }^{(6)}$.

Entre as atividades assistenciais desenvolvidas é possível observar que os enfermeiros se responsabilizam pelas ações relativas ao controle das condições dos pacientes internados (visita de enfermagem e avaliação do quadro clínico) e pelos cuidados aos pacientes graves. Além disso, dedicam-se à realização de procedimentos de maior complexidade técnica, tais como a realização de curativos, sondagem e punção venosa ${ }^{(6)}$. Nesta pesquisa, as menores médias de tempo despendidos em atividades assistenciais foram de 3,2 minutos para higiene e conforto, 5,1 minutos no auxílio ao paciente na alimentação e diferente do observado no estudo acima referido ${ }^{(6)}$. A confecção de curativos teve tempo médio de 5,2 minutos caracterizando um tempo médio baixo diante de outros procedimentos assistencias.

Podemos verificar que entre as atividades gerenciais, a realização da SAE ocupa a maior parte do tempo dos enfermeiros. É com o planejamento da assistência de enfermagem que o enfermeiro visa a definir, antecipadamente, as ações a serem implementadas pela equipe de enfermagem objetivando alcançar suas metas perante as necessidades dos pacientes/clientes. Com esse planejamento, o enfermeiro desencadeia as outras funções fundamentais da gerência, ou seja, a possibilidade de coordenar, controlar e avaliar o desempenho da equipe sob sua coordenação( ${ }^{(7)}$.

A função gerencial pode ser conceituada como um instrumento capaz de, política e tecnicamente, organizar o processo de trabalho com o objetivo de torná-lo qualificado e produtivo na oferta de uma assistência de enfermagem universal, igualitária e integral ${ }^{(5)}$.

$\mathrm{Na}$ Enfermagem, a gerência da unidade consiste na previsão, provisão, manutenção, controle de recursos materiais e humanos para o funcionamento do serviço, e a gerência do cuidado consiste no diagnóstico, planejamento, execução e avaliação da assistência, passando pela delegação das atividades, supervisão e orientação dos trabalhadores ${ }^{(5)}$. Assim os enfermeiros compreendem que gerenciar é cuidar e quando planejam, organizam, avaliam e coordenam, eles também estão cuidando ${ }^{(8)}$.

Observa-se um intenso movimento em direção a mudanças no perfil das habilidades esperadas do enfermeiro, havendo mudanças nas organizações, processos de trabalho e na documentação em saúde. No Brasil torna-se crescente a adoção de sistemas eletrônicos para as diversas atividades administrativas que dão suporte aos processos de trabalho assistenciais. A informatização da documentação dos processos assistenciais propriamente ditos exige profissionais de saúde preparados para direcionar e acompanhar mudanças que permitam alcançar resultados positivos para os processos de trabalho e também para a saúde das pessoas assistidas ${ }^{(9)}$. Isso se reflete no tempo de execução do trabalho, no ritmo em que ele acontece e como os profissionais estão preparados para atuar nesse ambiente, que exige habilidades que vão além da enfermagem.

Verificou-se que entre as atividades de apoio realizadas pelas enfermeiras, a comunicação com outros profissionais, familiares e pacientes é a atividade que demanda maior tempo para sua realização, porém pode-se afirmar que a comunicação é um ponto imprescindível para garantir que as atividades no gerenciamento de enfermagem ocorram de maneira eficiente e eficaz, já que proporciona ao enfermeiro se aproximar de sua equipe e demais profissionais com o intuito de compreender as atividades executadas, compartilhar ideias e visões, bem como criar interdependências para o desenvolvimento do trabalho através de equipes ${ }^{(10)}$.

Este estudo demonstrou que os enfermeiros consideraram a comunicação como importante ferramenta na prática profissional, pois é por meio dela que ocorre o entendimento da mensagem que está querendo ser transmitida e ela também promove um bom relacio- 
namento entre os profissionais e pacientes ${ }^{(11)}$. Embora os enfermeiros idealizem, em suas práticas, ações participativas e integralizadoras, há um consenso de que investimentos acerca de uma discussão mais profunda em torno de modelos comunicacionais ainda incipientes se fazem urgentes e necessário ${ }^{(12)}$.

Observando os resultados relativos ao tempo total consumidos pelos enfermeiros entre as diferentes atividades, podemos verificar que há uma prevalência para as atividades gerenciais. Estudos desenvolvidos em outras realidades também demonstram que os enfermeiros têm priorizado mais as atividades gerenciais ${ }^{(6,8-9)}$. Porém, deve-se considerar que o enfermeiro que atua com competência no gerenciamento de enfermagem garante boa qualidade da assistência ao paciente/cliente.

$\mathrm{Na}$ Enfermagem compete ao enfermeiro a coordenação da equipe, condução e viabilização do processo cuidativo e o mesmo necessita ter conhecimentos teóricos e vivências práticas ${ }^{(3)}$. Os profissionais de enfermagem devem conhecer a unidade em que atuam, dessa forma poderão identificar quais as necessidades do serviço para conseguirem balancear sua carga horária de acordo com a demanda do setor, independente se for mais assistencial, de gerência ou apoio, sempre levando em consideração a particularidade de cada unidade.

$\mathrm{O}$ enfermeiro, como gerente da assistência, deve ser capaz de identificar, analisar e conduzir as relações de trabalho sem que estas interfiram de forma negativa na assistência prestada aos clientes ${ }^{(3)}$. Quando se reflete sobre a gerência versus assistência há sempre uma polêmica, relativa ao distanciamento do enfermeiro do cuidado direto ao paciente, porém a gerência e o cuidado estão diretamente associados, uma vez que o enfermeiro ao gerenciar recursos em geral o faz focado para o processo assistencial e a fim de não se distanciar da qualidade da assistência. A qualidade da assistência tem como foco o paciente, mas deve-se levar em consideração: quem cuida deste paciente; qualidade técnica dos profissionais, quantos funcionários há para realizar a assistência; dimensionar recursos humanos, qual o material disponível para realização do cuidado; gerência de recursos materiais, qual a estrutura predial em que o cuidado acontece e o gerenciamento de recursos físicos.

Em síntese, o enfermeiro, em sua prática de gerenciamento, deve aprender a lidar com o conhecimento do seu grupo, do paciente, da instituição, com busca de uma mudança de paradigma. Este é um de seus desafios atuais.

\section{CONSIDERAÇÕES FINAIS}

Das atividades desenvolvidas, este estudo demonstrou que os enfermeiros têm se ocupado, mais frequentemente, com as atividades gerenciais que visam contemplar a assistência de enfermagem, principalmente, para atender as necessidades globais dos pacientes.

A contribuição da pesquisa se deu através do reconhecimento do papel desempenhado pelo enfermeiro durante a jornada de trabalho, o que possibilitou visualizar as lacunas entre as atribuições legais do enfermeiro e o processo de trabalho real. Também, favoreceu a gerência de enfermagem no diagnóstico estratégico de como a enfermagem investe o tempo durante a jornada de trabalho, a fim de dimensionar quantos profissionais seriam necessários para atender às necessidades da população atendida.

\section{REFERÊNCIAS}

1. Conselho Federal de Enfermagem.Código de ética profissional de enfermagem. Rio de Janeiro: COFEN; 2000 .

2. Conselho Federal de Enfermagem. Resolução COFEN no $272 / 2002$.[Internet] [acesso em 13 jul 2001]. Disponível: http://site.portalcofen.gov.br/node/4329.

3. Spagnol CA.(Re)pensando a gerência em enfermagem a partir de conceitos utilizados no campo da Saúde Coletiva. Cienc. saude coletiva. 2005;10(1):119-27.

4. Rocha SMM, Almeida MCP. O processo de trabalho da enfermagem em saúde coletiva e a interdisciplinaridade. Rev. latino-am enferm. 2000;8(6):96-101.

5. Horta WA. Processo de enfermagem.São Paulo: EPU; 1979.

6. Costa RA, Shimizu HE. Atividades desenvolvidas pelos enfermeiros nas unidades de internação de um hospitalescola.Rev. latino-am enferm. 2005;13(5):654-62.

7. Brasil. Lei n. 7498 de 25 de julho de 1986. Dispõe sobre a regulamentação do exercício da enfermagem e dá outras providências. DOU.1986 jul.26:seção 1; 9273-5.

8. Vaghetti H, Reis D, Kerber NC, Azambuja E, Fernandes G. Percepções dos enfermeiros acerca das ações administrativas em seu processo de trabalho. Rev. bras. enferm. 2004;57(3):316-20.

9. Trevizan MA. Enfermagem hospitalar: administração 
\& burocracia.Brasília (DF): UNB; 1988.

10. Trevizan MA. Estudo das atividades dos enfermeiroschefes de unidades de internação de um hospital-escola. Enferm. atual. 1980;3(14):17.

11. Corniani F, Galvão CM, Sawada NO. Liderança e comunicação: opinião dos enfermeiros responsáveis pelos serviços de enfermagem de um hospital governamental. Rev. esc enferm USP. 2000;34(4):347-53.

12. Spagnuolo RS, Pereira MLT. Práticas de saúde em enfermagem e comunicação: um estudo de revisão da literatura. Cienc. saude coletiva. 2007;12(6):1603-10. 